\title{
Las consecuencias de la formación del duopolio televisivo de Mediaset España y Atresmedia
} The consequences of the formation of the television duopoly of Mediaset España and Atresmedia

José Vicente García Santamaría, Universidad Carlos III - josevicente.garcia@uc3m.es

María José Pérez Serrano, Universidad Complutense de Madrid - mariajoseperezserrano@pdi.ucm.es

Gema Alcolea Díaz, CES Felipe II (UCM) - gema.alcolea@ajz.ucm.es

Resumen

El mercado publicitario español de medios convencionales se encuentra excesivamente concentrado. Mientras que en Internet los grandes players del sector -fundamentalmente, Google y Facebook- apenas dejan espacio para la supervivencia de las nuevas start ups periodísticas que han emergido desde el año 2010 hasta hoy, el mercado televisivo español está dominado claramente por el duopolio formado por Atresmedia y Mediaset España. Estas dos grandes plataformas acaparan cerca del 90\% de la contratación publicitaria de la televisión en abierto y, además, engloban a los únicos medios capaces de llegar en horario de prime time al conjunto de la audiencia en cualquier lugar de España.

Esta comunicación tiene como objetivo principal analizar el nivel de concentración publicitaria de este duopolio televisivo que, según su magnitud, puede suponer una amenaza a la supervivencia de otros proyectos periodísticos y televisivos; llegar a expulsar del mercado a otros operadores y crear, en definitiva, nuevas barreras de entrada en el mercado de medios convencionales.

\section{Palabras clave}

Concentración medios de comunicación; pluralismo informativo; contratación publicitaria; televisión.

Sumario

1. Introducción. 2. Hipótesis y objetivos de la investigación. 3. Metodología y enfoque. 4. La concentración del mercado de la televisión.

5. Conclusiones. 6. Referencias bibliográficas.

Abstract

The Spanish conventional media advertising market is excessively concentrated. While on the Internet the big players in the sector mainly Google and Facebook- leave little room for the survival of the new journalistic start-ups that have emerged since 2010, the Spanish television market is clearly dominated by the duopoly formed by Atresmedia and Mediaset España. These two major platforms account for nearly $90 \%$ of the advertising contracts on free-to-air television and, in addition, they are the only media capable of reaching the entire audience in prime time anywhere in Spain.

Keywords

Media concentration, media pluralism, advertising, contracting, television.

Summary

1. Introducción. 2. Hipótesis y objetivos de la investigación. 3. Metodología y enfoque. 4. La concentración del mercado de la televisión.

5. Conclusiones. 6 . Referencias bibliográficas. 


\section{Introducción}

Los procesos de integración vertical y horizontal han sido una estrategia recurrente de los grandes grupos de comunicación desde mediados de la década de los noventa, tanto para ampliar mercados, capitalizar los desarrollos tecnológicos o aplicar economías de escala (Chan- Olmsted, 1998). Pero este tipo de integraciones conlleva altos costes de capital y reduce la competencia (Anderson, Bell y Shirky, 2013).

Al mismo tiempo, las operaciones de fusión o absorción entre compañias que operan en el mismo sector de actividad permiten no sólo estar presentes en toda la cadena de valor, sino también reducir el número de participantes en un mercado e incrementar la rentabilidad de la compañía resultante (Peltier, 2004). Además, en algunos países, estos procesos conllevan importantes incentivos fiscales para la matriz.

Mientras que las operaciones de integración vertical se han saldado -en el ámbito internacional- con casos de éxito al lograr que los grandes grupos de comunicación (News Corp., Walt Disney Co.) o empresas de cable (Comcast), se hayan expandido por todo el mundo y generado valor para sus accionistas ${ }^{1}$, las estrategias de integración horizontal, que permiten incrementar sustancialmente la cuota en un mercado, han gozado, sin embargo, de desigual resultado.

En el caso español, el ejemplo más evidente ha sido la adquisición en el año 2007 del grupo Recoletos por parte de Unedisa por un importe de 1.100 millones de euros. La empresa de RCS Mediagroup aspiraba, así, a convertirse en líder de la prensa en España y tomar posiciones en otros mercados como el radiofónico y televisivo. Pero la consecuencia evidente es que, el hecho de apostar por un sector en declive como el de la prensa escrita y que no encuentra un modelo de negocio rentable en la era de Internet, ha lastrado las cuentas del grupo y frustrado sus expectativas de crecimiento.

En el sector de la televisión privada en abierto, por el contrario, las recientes absorciones de Cuatro por Telecinco y de La Sexta por Antena 3 han mejorado sustancialmente las cuentas de resultados de los grupos absorbentes y les han permitido, en un corto espacio de tiempo, ganar cuotas de audiencia y dominar, de manera amplia, la contratación publicitaria (García Santamaría, 2013). Unos efectos que se traducirán -una vez superada la crisis económica- en importantes incrementos en la facturación de ambas plataformas.

Cuadro no 1: Mediaset España y Atresmedia (Datos consolidados - año 2012)

\begin{tabular}{|c|c|c|c|c|c|}
\hline AคิO 2012 & $\begin{array}{l}\text { INGRESOS DE } \\
\text { EXPLOTACION } \\
\text { (imiles } 6)\end{array}$ & $\begin{array}{c}\text { RESULTADOS } \\
\text { ORDINARIOS ANTES } \\
\text { DE IMPUESTOS } \\
\text { (miles } 6 \text { ) }\end{array}$ & $\begin{array}{l}\text { TOTAL } \\
\text { ACTIVo } \\
\text { (miles } 6)\end{array}$ & $\begin{array}{l}\text { FONDOS } \\
\text { PROPIOS } \\
\text { (imilas } 6)\end{array}$ & $\begin{array}{l}\text { MÚMERO DE } \\
\text { EMPLEADOS }\end{array}$ \\
\hline $\begin{array}{l}\text { ATRESMEDIA CORPORACION } \\
\text { DE MEDIOS DE } \\
\text { COMUNICACION, S.A. }\end{array}$ & 741.163 & 11.904 & 1.173 .789 & 396.202 & 1.705 \\
\hline $\begin{array}{l}\text { MEDLASET ESPANA } \\
\text { COMUNICACION, SA. }\end{array}$ & 806727 & 52.392 & $1,767,160$ & $1,420,899$ & 1.360 \\
\hline
\end{tabular}

Fuente: SABI (Sistema de Análisis de Balances Ibéricos)/Elaboración propia

Ahora bien, a la consecución de un mercado más eficiente se le opone igualmente y, en muchas ocasiones, una excesiva concentración sectorial que llega a acaparar una parte importante del mercado dando lugar a la formación de "oligopolios imperfectos" o incluso de duopolios. En este caso, los conglomerados resultantes pueden imponer sus condiciones en el mercado, generando incluso "cuellos de botella" para los grandes anunciantes publicitarios que se ven obligados a contratar sus campañas con estas plataformas si desean llegar a un amplio target para sus productos en toda España y en horario de prime time.

Las autoridades de la competencia -en este caso la Comisión Nacional de la Competencia (CNC)- que en su día autorizaron, supeditadas al cumplimiento de ciertas condiciones, la absorción de Cuatro por Telecinco y de La Sexta por Antena 32, serían la última

\footnotetext{
${ }^{1}$ No obstante, también ha habido un saldo de operaciones fallidas, como ha sido el caso de la francesa Vivendi tras el control de NBC.

2 Las condiciones impuestas por la CNC a esta absorción fueron finalmente modificadas por el Consejo de 
frontera para hacer frente al dominio abrumador de dos empresas que controlan de manera clara el mercado de la televisión en abierto en España, y que cuentan, además, con derivaciones en la televisión de pago y en la producción y distribución de contenidos audiovisuales. Una situación que apenas encuentra parangón en el conjunto de países de la OCDE (Organización para la Cooperación y Desarrollo Económicos), a excepción de Puerto Rico y de Uruguay.

Existe, además, una consecuencia de estos procesos de crecimiento externo que preocupa por encima del resto, y es que una elevada concentración en los media puede poner en peligro el necesario pluralismo informativo y las elecciones independientes de la audiencia. Almirón et al., (2012), que han estudiado el funcionamiento de diferentes Consejos Audiovisuales, consideran esencial el papel de estas instituciones para preservar unos medios plurales en un país democrático.

Pero, como también señala Doyle (2003, p. 11), la definición de pluralismo hace referencia tanto a la diversidad de la propiedad como a los contenidos distribuidos. Dicho de otro modo, en estructuras de mercado más concentradas se corre el peligro de que los players dominantes restrinjan el número de voces a la hora de emitir informaciones, opiniones y valores (Faustino,

2010), siendo así lesivas para el pluralismo de las ideas. Autores como George (2007) han encontrado evidencias empíricas entre el grado de concentración y la variedad de los contenidos temáticos en un sector como el de la prensa norteamericana.

El pluralismo en los medios de comunicación es un valor fundamental del sistema democrático (Tomás Olalla, 2004), que se manifiesta en la garantía de acceso de los ciudadanos a una pluralidad de medios y contenidos independientes, y es un concepto protegido por la Unesco (2005). Por otra parte, la Comisión Europea en su Libro Verde de 1992 ha respaldado la libre circulación de la información. Y el Parlamento Europeo (2008) ha indicado que el exceso de concentración en los media puede favorecer tendencias monopolísticas en el mercado.

Por su parte, el informe del Consejo de Europa sobre Concentraciones Transnacionales de Medios en Europa (2004) indica que las autoridades de la competencia solamente están interesadas en asuntos de competencia económica, cuando en este ámbito es muy importante la competencia periodística para asegurar el pluralismo informativo (Fernández, 2013).

\section{Hipótesis y objetivos de la investigación}

La hipótesis de partida hace referencia a que la modificación de la cadena de valor televisiva, con la consolidación del duopolio formado por Atresmedia y Mediaset España, puede llegar a la formación de cárteles que restrinjan la competencia en la contratación publicitaria de los denominados "medios convencionales". Es en esta consecuencia de la concentración en la que centraremos el trabajo, sin entrar en el estudio de otras posibles, como la disminución de la competencia en los mercados de producción audiovisual y en los de adquisición de derechos de difusión de contenidos.

Esta proposición conjetural se completa al subrayar que el dominio de las dos grandes plataformas televisivas puede jugar igualmente un papel relevante en la supervivencia de las televisiones públicas y privadas en abierto, e incluso en el futuro de las empresas radiofónicas y de las publicaciones impresas y online.

\section{Metodología y enfoque}

Metodológicamente, la Comunicación parte de la economía política de la comunicación (EPC) y del análisis de la propiedad y concentración de los media. Como es sabido, la EPC proporciona un marco teórico integrado (Mosco, 1996) que además de ocuparse de las relaciones de poder se centra especialmente en la propiedad y concentración de los medios de comunicación. Se parte de la literatura académica de la referencia sobre estos temas a nivel nacional e internacional.

En España existe un conjunto de académicos que vienen ocupándose desde la década de los noventa hasta hoy día, ya sea con carácter más general y, en algunos casos, hasta tangencial, de los procesos de concentración. En este grupo destacan: Alcolea (2003), Campos Freire (2012), García Castillejo (2009), García Santamaría y Fernández-Beaumont (2012), Llorens (2010), Miguel de Bustos (2007), Pérez Gómez (2002), Rodríguez Pardo (2005), Segovia (2001) o Sánchez-Tabernero (2010). Al mismo tiempo, el estudio de la economía televisiva y/o de las industrias culturales ha sido también objeto de atención preferente por autores como Arnanz (2002), Álvarez Monzoncillo (2011), Artero (2008), Bustamante (2009) o Zallo (2011), entre otros.

Una buena parte de estos autores centran su atención en los procesos de concentración de la propiedad (situaciones monopolísticas u oligopolísticas), capaces de alterar tanto la libre competencia como el pluralismo informativo. No obstante,

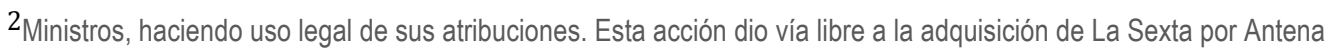

23 , puesto que estos operadores habían comunicado públicamente que no llevarían a cabo dicho proceso de mantenerse las condiciones impuestas originariamente por la autoridad de la competencia. La CNC consideraba que las condiciones impuestas a este segundo proceso de absorción eran diferentes y más restrictivas que en el anterior caso, por la diferente situación en la que se encontraba ya el mercado, paliando los posibles efectos de la concentración, lesivos para los intereses de muchas de las partes afectadas. Véase Alcolea y Pérez Serrano (2013).
} 
queda por estudiar en profundidad los efectos de la inversión publicitaria sobre el pluralismo (Aguirre, González, 2012) y las consecuencias que se han derivado de las estrategias de concentración para los media españoles.

Como fuentes de referencia, hemos recurrido también a los informes y documentos de la Comisión del Mercado de las Telecomunicaciones (CMT) y de la Comisión Nacional de la Competencia (CNC); cifras de contratación publicitaria y de audiencias de Infoadex, Barlovento; los balances económico-financieros de Atresmedia y Mediaset España y la legislación en la materia.

En esta comunicación se han examinado también los niveles de concentración de las televisiones en abierto; la evolución de la contratación de los medios convencionales entre los años 2007 y 2012, y de las audiencias televisivas en el mismo período de tiempo. Por otra parte, se ha tenido en cuenta la incidencia de la legislación y las políticas aplicadas por los partidos en el gobierno sobre la conformación de la televisión en España, así como el mercado de contenidos.

En cuanto a los datos de inversión de publicidad hemos procedido a analizar la inversión realizada en televisión y, por ende, en las plataformas objeto de estudio, por parte de las diez principales empresas en el ranking de inversión publicitaria. En este análisis debe tenerse en cuenta que la selección de la muestra se basa en el escalafón que la empresa Arce Media hace de la inversión publicitaria a través de las marcas y creatividades que más invierten, pero hemos optado, como criterio básico, el "valor en miles de euros", por estar más próximo a las características de nuestro objeto de estudio y por eliminar el factor "futuro". Esto hace que el ranking varíe de acuerdo con el cuadro que señalamos a continuación y a las observaciones que se establecen.

\begin{tabular}{|c|c|c|c|c|c|}
\hline \multicolumn{2}{|c|}{$\begin{array}{l}\text { CRITERID VAL OR [N" } \\
\text { WACE NEDWi }\end{array}$} & \multicolumn{2}{|c|}{$\begin{array}{l}\text { CAITERIO VIL OS EN } \\
\text { MILSS OE }\end{array}$} & \multirow{2}{*}{ MaEA } & \multirow{2}{*}{$\begin{array}{l}\text { OESEFWCIONES } \\
\text { LEEWA SEWUES }\end{array}$} \\
\hline 1 & FUND FEPSOL & 1 & TPRLMATh & & \\
\hline 2 & TRPYNof & 2 & FUNO AGPAL & repsol & 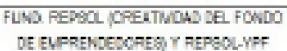 \\
\hline 1 & MUTLA EOAPIEVI & \multirow{2}{*}{3} & WDSTIBR & 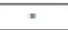 & \\
\hline 4 & MOWEThE & & Bowr & 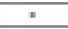 & 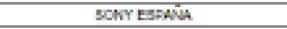 \\
\hline & BWY' & 4 & WUTUA HASLLE' &. & \\
\hline \multirow[t]{2}{*}{5} & $4 a$ & \multirow[t]{2}{*}{5} & La & - & LA ELEOTFONGS \\
\hline & DW0 & & OND & $=$ & \\
\hline 5 & FEUESOT & 6 & Ghishate & - & FFALDE TELECOM \\
\hline 7 & WeBREE & $T$ & PELGEOT & $=$ & \\
\hline$\pi$ & orance & 8 & WEACEDES & 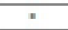 & UEFGEDEE EBN? \\
\hline 9 & ULEALNCCS & 8 & NESFAESD & 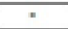 & RESTLE KESPFESSO \\
\hline 18 & Mtgpmesso & 10 & ML_. Ayumatos &. & \\
\hline
\end{tabular}

Cuadro n² 2: Ranking de inversión publicitaria en televisión según criterios (octubre 2013)

Fuente: Arce Media-Dragón/Elaboración propia

Por otro lado, se ha procedido a comparar la inversión en el prime time de las principales cadenas de Atresmedia y Mediaset. Cronológicamente, hemos seleccionado octubre de 2013 porque es el mes completo más cercano al cierre de la investigación. Así como para la comparación de la inversión en el prime time de las principales cadenas de Atresmedia y Mediaset, hemos seleccionado el día 21 de octubre de 2013. La justificación del tercer lunes del mes de octubre está vinculada a diversas circunstancias que concurrieron ese día:

- El primer día de la semana es uno de los que presenta mayor consumo televisivo y, además, una jornada que quedaba "libre" en la cadena de Atresmedia.

- El día 21 de octubre de 2013 se estrena "El tiempo entre costuras", en Antena 3.

- Su comienzo se "convirtió en el mejor debut de ficción nacional de Antena 3 en los últimos 12 años y de todas las series de televisión desde 2005" (Vertele, 2013).

- La nueva ficción de época lleva al mínimo al talent show "La Voz" (20,2\% de cuota de pantalla), en Telecinco, y a la serie 'Isabel' (14,8\% de cuota de pantalla y 3.023 .000 - mínimo histórico-), en TVE1. Ambos programas en la misma franja horaria.

- El minuto más visto de ese día corresponde a la ficción de Antena 3, a las a las 23:46 horas, con 5.703.000 espectadores y un $31,6 \%$ de cuota de pantalla.

- "El tiempo entre costuras" y "La Voz" van dirigidos a un target de espectadores complementario. Pero 
"Isabel" y la ficción basada en la novela de María Dueñas lo comparten.

- Boomerang TV, productora de "El tiempo entre costuras" es la misma que la de "La Voz", con lo cual podemos decir que dos cadenas compiten contra la cadena nacional con dos productos de la misma "fábrica".

Es necesario tener en cuenta que somos conscientes de que el prime time es una franja horaria más abierta que el espacio en la parrilla que ocupa "El tiempo entre costuras" y que en España este singular -y, quizás, tradicional- prime space es más largo en que otros países (aquí puede abarcar desde las $21,00 \mathrm{~h}$. a pasadas las $24,00 \mathrm{~h}$.), pero nos parecía significativa la inversión publicitaria en dicho momento del día, teniendo en cuenta, sobre todo, que desde Ley $8 / 2009$, de

28 de agosto, de financiación de la CRTVE, TVE1 carece de publicidad al uso. La base cuantitativa de estos hechos la plasmamos como sigue.

Cuadro $n^{0} 3:$ Datos de audiencia. Cuota de pantalla o share del 21 de octubre 2013

\begin{tabular}{|c|c|c|c|c|c|}
\hline & GRUPOS & MEDIA DEL DIA & $\begin{array}{l}\text { MEDIA DEL MES } \\
\text { (HASTA EL 21 DE } \\
\text { OCTUDRE DE 2013) }\end{array}$ & CONTENIDO & SHARE MEDIO \\
\hline 1 & ATRESNEDIA & $31,2 \%$ & $298 \%$ & "E lierpo entre costuras" & $25,5 \%$ \\
\hline 2 & MEDUASET ESPAKA & $30,1 \%$ & $28,9 \%$ & "La vaz" & $18,9 \%$ \\
\hline 3 & RTVE & 15, 日, & $16.5 \%$ & "lsabel" & $13,5 \%$ \\
\hline
\end{tabular}

Fuente: Kantar Media/Elaboración propia

\section{La concentración del mercado de la televisión}

Las televisiones privadas concentran el $50 \%$ de la publicidad total en España y cerca del $\mathbf{9 0 \%}$ de la contratación en televisión (Infoadex, 2013, Barlovento Comunicación, 2013). Aunque la tendencia general -al igual que en otros países de la UE y Estados Unidos- es la fragmentación de las audiencias televisivas, la constitución de plataformas televisivas, derivadas también de la apuesta por la Televisión Digital Terrestre (TDT) y del mapa de concentraciones, compensa esta pérdida de telespectadores en el canal principal con los que la plataforma logra captar en el resto de canales que operan en la TDT.

Los datos del mes de octubre de 2013 (Barlovento Comunicación, 2013) reflejan la siguiente distribución de audiencias: Antena 3 cuenta con el $13,6 \%$ del total de la audiencia, pero su plataforma Atresmedia dispone del $29 \%$. En cuanto a Telecinco, posee el $14,1 \%$, y Mediaset España el $29,5 \%$. Y, CRTVE se sitúa en $16,5 \%$.

Resulta, por tanto, fundamental, la negociación de la emisión de publicidad a través de la plataforma televisiva, si bien la antigua CNC (ahora CNMC -Comisión Nacional de los Mercados y la Competencia-) había prohibido la comercialización conjunta de publicidad entre Cuatro y Telecinco y entre La Sexta y Antena 3. Pero, tras la retirada de TVE de la contratación publicitaria y de las pobres audiencias que consiguen el resto de los concurrentes en este mercado, la única posibilidad que poseen los grandes anunciantes de llegar en horario de prime time al conjunto de los hogares de toda España es a través de las dos grandes plataformas televisivas: Atresmedia y Mediaset España. El hecho de la llegada, tras el apagón digital y la llegada de la TDT, de nuevos competidores al mercado televisivo y la existencia de 24 canales privados de ámbito estatal más los ocho de la CRTVE, no ha significado una modificación del marco competencial televisivo. Más bien todo lo contrario, puesto que en la televisión en abierto, no existe alternativa publicitaria fuera del duopolio.

Es cierto también que, tras la recesión del mercado publicitario a comienzos de la década de los noventa (Artero, 2008), el final de esa década y los ocho primeros años del siglo XXI se caracterizaron por un incremento sustancial de la publicidad contratada por las grandes empresas: al relanzamiento del consumo de las familias se le unieron las facilidades crediticias otorgadas por bancos y cajas; el desarrollo del mercado automovilístico y también la emergencia de las compañías de telecomunicaciones, y las compañías punteras en estos sectores recurrieron a enormes campañas de marketing que utilizaron a las grandes cadenas para llegar de manera efectiva a su público objetivo. Mientras que la estrella de CRTVE declinaba hasta dotarla en el año 2009 de un nuevo y restrictivo marco financiero (Ley 8/2009, de 28 de agosto, de financiación de la Corporación de Radio y Televisión Española), y las autonómicas públicas crecieron hasta que vieron restringidos sus presupuestos de funcionamiento, las grandes cadenas privadas mejoraron sustancialmente su cuenta de resultados, incluso Telecinco se permitía anunciar que había pagado a sus accionistas más de 1.500 millones de euros en dividendos desde su creación (buena parte de ellos, obtenidos desde finales de los noventa).

Al final, sin embargo, y tras la llegada de la recesión de la economía española se pusieron en evidencia cuatro hechos importantes: 1) El mercado publicitario televisivo español era tan estrecho que sólo podía permitir la supervivencia de cinco canales de televisión en abierto en un marco de expansión. 2) No se valoraron los efectos de una competencia mayor tras la creación de Cuatro y La Sexta. 3) No se tuvieron tampoco en cuenta las amenazas que se cernían sobre el mercado televisivo: mayor fragmentación de audiencias; encarecimiento de los contenidos Premium, sobre todo los derechos de retransmisiones deportivas; la competencia, aunque incipiente, de la TV IP y de la televisión de pago, y los nuevos hábitos de consumo televisivo. 4) No existe ningún otro país en la OCDE -salvo los casos de Puerto Rico y Uruguay (Lanza y Buquet, 2011)- donde un solo grupo televisivo detente el $30 \%$ del mercado publicitario de medios convencionales y más del $40 \%$ del televisivo.

Todos estos imponderables, trajeron como consecuencia la modificación de la cadena de valor televisiva y, al igual que sucedió con la prensa, y en menor medida con la radio, la mayor parte de las cadenas y de los grupos de comunicación a los 
que pertenecían no hallaron modelos de negocio viables (Bustamante, 2009).

Por lo que respecta a la contratación publicitaria del ejercicio $2012^{3}$, con datos de Infoadex, las dos grandes plataformas televisivas alcanzaron más del $87 \%$ del total, con una cifra de negocio de 1.575 millones de euros. Baste decir que entre las televisiones autonómicas y los canales de pago sumaron 170 millones de euros, apenas el $11 \%$ de la factura publicitaria.

Distribución por medios que se reproduce claramente en la muestra analizada, como hemos dicho correspondiente al mes de octubre de 2013, en cuanto a las diez marcas con mayor inversión según el ranking anteriormente expuesto (cuadro 4).

Cuadro nº 4: Inversión publicitaria en medios en octubre de 2013 (miles de $€$ )

\begin{tabular}{|c|c|c|c|c|}
\hline \multicolumn{2}{|r|}{ MARCAS } & \multirow{2}{*}{$\begin{array}{l}\text { VALOR DE LA } \\
\text { INVERSION EN } \\
\text { TELEVISION } \\
\text { 6. } 123,37\end{array}$} & \multirow{2}{*}{$\begin{array}{l}\text { INEERSION EN } \\
\text { MEDIOS } \\
\text { IMPRESOS } \\
\text { 0,00 }\end{array}$} & \multirow{2}{*}{$\begin{array}{l}\text { INVERSION EN } \\
\text { RADIO } \\
\\
0,00\end{array}$} \\
\hline 1 & TRIPADVISOR & & & \\
\hline 2 & REPSOL & $6.727,43$ & 974,43 & 852,00 \\
\hline 3 & MOVISTAR & $16.184,80$ & 4220,21 & $2.448,83$ \\
\hline & SONY & $18.769,12$ & 2289,12 & 406,20 \\
\hline 4 & MUTUA MADRILENA & $7.397,17$ & 56,05 & $1.366,38$ \\
\hline \multirow{2}{*}{5} & 10 & $7.614,05$ & 280,55 & 50,14 \\
\hline & ONO & $6.315,30$ & 0,80 & 0,00 \\
\hline 6 & ORANGE & $11.980,38$ & 1865,69 & 0,00 \\
\hline 7 & PEUGEOT & $7.188,70$ & $2.506,89$ & $1.770,20$ \\
\hline 8 & MERCEDES & $3.453,52$ & 98,12 & 924,94 \\
\hline 9 & NESPRESSO & $4,116.23$ & 0,00 & 0,00 \\
\hline & MIL ANUNCIOS & $4,474,30$ & 788,89 & 0,00 \\
\hline TOT & & $100,372,36$ & $13.065,56$ & $7.908,70$ \\
\hline
\end{tabular}

Fuente: Arce Media-Dragón/Elaboración propia

La tendencia hacia una concentración todavía mayor del duopolio televisivo parece clara: si en el año 2011 concentraban el $85,4 \%$ del mercado, en el año 2012 habían sobrepasado el 87\%, y las cifras a septiembre de 2013 llegan incluso al 89\%. Así, del total de la inversión publicitaria en medios convencionales, exceptuando la publicidad exterior e Internet, la contratación televisiva supone el $60 \%$ del total, una tendencia que tiende a acrecentarse en cada ejercicio.

Aunque de manera empírica, y con datos difíciles de contrastar dada la carencia de investigaciones de mercado en este sentido, no resulta aventurado suponer que, en el período que abarca de 2007 a 2012, una parte de casi el $60 \%$ que disminuyó la publicidad en prensa, puede haber ido a parar a las televisiones en abierto -y a Internet-. La profunda crisis que ha atravesado la prensa, la disminución del número de ejemplares vendidos y la alternativa de medios online ha podido inducir a los grandes anunciantes a concentrar sus esfuerzos en las dos grandes plataformas televisivas, precisamente por su gran capilaridad territorial y sus audiencias concentradas. A pesar, incluso, de que como han denunciado reiteradamente, las tarifas hayan subido más de un $20 \%$ en plena crisis. Que las plataformas acaparan una parte mayoritaria de la inversión publicitaria en la televisión en abierto queda patente tras el estudio de la distribución de la misma, como se muestra con los datos del mes de octubre. El volumen de inversión de las principales marcas oscila desde el $81,6 \%$ hasta un mínimo del $11,23 \%$, pero el porcentaje total de inversión supera el $58 \%$ para las creatividades estudiadas.

Cuadro $n^{0}$ 5: Inversión publicitaria en televisión en octubre de 2013 (miles de €)

3 Aunque esta Comunicación se ha cerrado antes de la conclusión de 2013, todo parece indicar que la contratación publicitaria en televisión repuntará en este ejercicio, mejorando así la cuenta de resultados de las dos grandes plataformas. En los nueve primeros meses del año 2013, la inversión publicitaria dirigida a medios convencionales disminuyó el 12,6\% respecto al mismo periodo de 2012, mostrando una cifra de 2.548,9 millones de euros, frente a los 2.917,6 millones del año anterior (Infoadex, 2013). 


\begin{tabular}{|c|c|c|c|c|c|}
\hline \multicolumn{2}{|r|}{ MARCAS } & $\begin{array}{l}\text { VALOR DE LA } \\
\text { INVERSION EN } \\
\text { TELEVISION }\end{array}$ & $\begin{array}{l}\text { TOTAL DE LA } \\
\text { WNERSION EN } \\
\text { ATRESUEDIA Y } \\
\text { MEDIASET } \\
\text { ESPANA }\end{array}$ & 8 & $\begin{array}{l}\text { TOTAL EN EL } \\
\text { RESTO DE } \\
\text { TELEVISIONES }\end{array}$ \\
\hline 1 & TRAPADVSOR & 6129,37 & 450644 & 81,60 & $1,126.94$ \\
\hline 2 & REPSOL & 6727,43 & 756,64 & 11,23 & 5971,80 \\
\hline \multirow{2}{*}{3} & MOVSTAR & $16.184,80$ & 7958,13 & 49,14 & 8.23167 \\
\hline & SONY & 18789,12 & 11324.61 & 60,33 & 7.454 .51 \\
\hline 4 & MUTUA MADRILENA & $7.399,17$ & $5,256,52$ & 78,75 & 1.57168 \\
\hline \multirow{2}{*}{5} & LG & 7614,05 & 4900,17 & 64,75 & 288389 \\
\hline & ONO & 6315,30 & $3.867,74$ & 61,24 & 24475 \\
\hline 6 & ORANGE & 11908,38 & B.BDB, 75 & 73,48 & $3.179,60$ \\
\hline 7 & PEUGGEOT & $7.189,70$ & 358299 & 49,84 & 3605,72 \\
\hline 8 & MERCEDES & 3451,52 & $2.129,34$ & 61,60 & $1.324,19$ \\
\hline 9 & NESPRESSO & $4.116,23$ & 1.919,89 & 46,64 & 2196,30 \\
\hline 10 & ML_ ANUACIOS & 4474,30 & 245297 & 54,82 & 2021,39 \\
\hline \multicolumn{2}{|c|}{ TOTAL } & 100372,35 & $58,557,19$ & 58,34 & $41,815,18$ \\
\hline
\end{tabular}

Fuente: Arce Media-Dragón/Elaboración propia

Asimismo, se puede apreciar de los datos estudiados la distribución en dicha publicidad en ambas plataformas. En los cuadros 6 y 7 se ofrece un desglose de la inversión publicitaria en Atresmedia y Mediaset España, respectivamente. Por otro lado, en el cuadro número 8 se puede apreciar la inversión de estas marcas en el prime time en las principales cadenas de las plataformas.

Cuadro $n^{0}$ 6: Inversión publicitaria en Atresmedia en octubre de 2013 (miles de $€$ )

\begin{tabular}{|c|c|c|c|c|c|c|c|c|c|c|}
\hline & \multirow{2}{*}{ MARCAS } & \multirow{2}{*}{$\begin{array}{l}\text { INVERSION } \\
\text { TELEVISION }\end{array}$} & \multicolumn{2}{|c|}{ Antena 1} & \multicolumn{2}{|c|}{ La Saxta } & \multicolumn{2}{|c|}{ Plataforma } & \multicolumn{2}{|c|}{$\begin{array}{l}\text { Imarsidn total an } \\
\text { Atrosmedia }\end{array}$} \\
\hline & & & Importe & $\begin{array}{l}\text { 95 sobre of } \\
\text { valor tetal }\end{array}$ & Imports & $\begin{array}{l}\text { 5. sober al } \\
\text { valor total }\end{array}$ & Imparts & $\begin{array}{l}\text { 4) sobere el } \\
\text { valur tobal }\end{array}$ & Imparte & $\begin{array}{l}\text { So sobre el } \\
\text { valor total }\end{array}$ \\
\hline 1 & TRIPAOUSOR & $6.123,37$ & 1.570 .27 & 25,646 & 287,05 & 367 & 117,16 & 1,914 & 64745 & $27,56 \%$ \\
\hline 2 & REPSOLL & $6.727,43$ & 457,60 & 0,790 & 0,00 & $0 \%$ & 375 & DEN & 460,75 & \\
\hline \multirow{2}{*}{$s$} & MOVSTAR & $16.184,100$ & 2451,76 & $15,15 \%$ & $1.218,8 \mathrm{~B}$ & 7,53 & 495.55 & $2,60 \%$ & 2 bar 32 & $17,84 \mathrm{~s}$ \\
\hline & Soky & $18.784,12$ & $3.518,11$ & $16,72 \%$ & 1700,90 & 9,45 & 177,14 & $0,94 \mathrm{k}$ & 3606.25 & $19,67 \%$ \\
\hline 4 & $\begin{array}{l}\text { MUTUA } \\
\text { MADRILEA }\end{array}$ & $7,390,17$ & 1987.96 & sis & 30.94 & $5.19 x$ & 12695 & $1.7 \%$ & 2.114 .90 & 20450 \\
\hline \multirow{2}{*}{5} & Lo & $7,514,05$ & 1281,36 & 16506 & 124,75 & $163 \%$ & 1418.12 & $1.95 \%$ & $1,439,48$ & 13.945 \\
\hline & ONO & $6.315,30$ & 780,3 & $12,66 \%$ & 990,10 & $15,73 \%$ & 65471 & $10,37 \%$ & $1.454,04$ & $2,02 \%$ \\
\hline 6 & OAANGE & 11, ses 35 & 26721 & 2235 & 67,06 & 565 & 1492 & $4.35 \mathrm{~S}$ & 300.14 & 2575 \\
\hline 7 & PEUGEOT & 7.16070 & 961,3 & $13,37 \%$ & 79,76 & 11,118 & 302.95 & 5,428 & 1.31 .26 & $1300 \%$ \\
\hline 8 & MERLEEDES & 3.45352 & 800.5 & 2,248 & 452,77 & 13,118 & 85.71 & $7,40 \%$ & 1.osén. 26 & 30,644 \\
\hline 9 & NESPFESSO & $4.116,23$ & $676,6 \pi$ & $16,44 \%$ & 457,80 & $11,85 \%$ & 21372 & $5,10 \mathrm{~m}$ & 820.98 & $21,69 \%$ \\
\hline 10 & MiL AnUNCIÓs & $4,474,30$ & 900,35 & 21,848 & $1.04,76$ & $28,17 \mathrm{k}$ & 24667 & 5.665 & 1.219 .02 & $27,24 \%$ \\
\hline T01 & TaL & 100.372 .37 & $16,163,69$ & 18,10 青 & 9311,53 & $926 \%$ & $1.296,39$ & $3.28 \%$ & $21,460,28$ & $21,3 \mathrm{Ex}$ \\
\hline
\end{tabular}

Fuente: Arce Media-Dragón/Elaboración propia

Cuadro n 7: Inversión publicitaria en Mediaset España en octubre de 2013 (miles de $€$ ) 


\begin{tabular}{|c|c|c|c|c|c|c|c|c|c|c|}
\hline \multirow{2}{*}{\multicolumn{2}{|c|}{ MARCAS }} & \multirow{2}{*}{$\begin{array}{l}\text { IWVERSION } \\
\text { TELEVISIOW }\end{array}$} & \multicolumn{2}{|c|}{ Telacinces } & \multicolumn{2}{|c|}{ Cueiro } & \multicolumn{2}{|c|}{ Platalorma } & \multicolumn{2}{|c|}{$\begin{array}{l}\text { Inversión total en } \\
\text { Modiased Eapaina }\end{array}$} \\
\hline & & & Imporiat & $\begin{array}{l}\text { \% sobre of } \\
\text { valor total }\end{array}$ & Importa & $\begin{array}{l}\text { Yaobre ef } \\
\text { valar total }\end{array}$ & Imperte & $\begin{array}{l}\text { Pin solbet et } \\
\text { Walor tatal }\end{array}$ & Imperte & $\begin{array}{l}\% \text { sobre al } \\
\text { valor total }\end{array}$ \\
\hline 1 & TRRADVSOR & 123.37 & & & & a.28: & nut mo & & & \\
\hline 2 & REPSOL & 27,43 & 2 & & 124,90 & 360 & & & & \\
\hline & WOMSTAR & $16.184,80$ & $3.452,02$ & $21,21 \%$ & 2387,94 & $14,45 \%$ & $1.605,7$ & $10,09 \%$ & 5066,81 & $1,20 \%$ \\
\hline & SONY & $16.769,12$ & $4.77,27$ & $25, \omega 4$ & 305610 & $16,46 x_{3}$ & 2606,10 & $15,25 \%$ & 7359,96 & $40,6 \%$ \\
\hline & $\begin{array}{l}\text { MUTUA } \\
\text { MADRILEAA }\end{array}$ & 7397,17 & $2.441,01$ & $33,00 \%$ & 660,4 & $9.26 \mathrm{~m}$ & $1.294,67$ & $17,16 \times$ & $3.710,02$ & $60,16 \%$ \\
\hline 5 & LG & 1614,06 & 1999,80 & 896 & $1.075,11$ & 14,128 & $1.290,60$ & 1965 & $4=0,69$ & $5,65 \%$ \\
\hline & ONO & 6315,30 & 1.4543 & 105: & 55,8 & 9,430 & 6593 & 3,1900 & 413,71 & 37 \\
\hline 6 & ORANGE & 19683 & 939.56 & 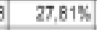 & 1233,15 & $10.20 \%$ & 270 & $1896 \%$ & 5606.61 & $697 \%$ \\
\hline 7 & PEUGEOT & $7.160,70$ & 12280 & $17,07 \%$ & 1672,8 & $2,2 \pi$ & $1.004,61 \mid$ & $139 \%$ & 2251,70 & $31,04 \%$ \\
\hline 自 & MEACEDES & $3.451,12$ & 67,52 & 196 & 757,40 & 21,80 & 31,5 & $11,24 \%$ & $10 \% 1,00$ & $31,01 \%$ \\
\hline 9 & NESPRESSO & 4.1167 & 59,73 & $14,48 \%$ & 958 & 217 & 4357 & $105 \mathrm{~s}$ & 10690 & $2501 \%$ \\
\hline & MLANUNCIOS & $4,474,30$ & $1,034,48$ & $23,12 \%$ & 912.58 & $20.40 \%$ & 190,6 & $4,46 \%$ & 120,95 & $27,5 \mathrm{~K}$ \\
\hline & DTAL & $104.32,37$ & $22.726,07$ & $22,84 \%$ & $13.660,45$ & $13,0 \%$ & 1437084 & $14,32 \%$ & 770061 & $36.96 \%$ \\
\hline
\end{tabular}

Fuente: Arce Media-Dragón/Elaboración propia

Cuadro n 8: Comparación de la misma inversión en el prime time de las principales cadenas de ambos grupos (miles de $€$ )

\begin{tabular}{|c|c|c|c|c|c|c|}
\hline \multirow{2}{*}{\multicolumn{2}{|c|}{ MARCAS }} & \multirow{2}{*}{$\begin{array}{l}\text { INVERSION EN } \\
\text { TELEVISIÓN }\end{array}$} & \multicolumn{2}{|c|}{$\begin{array}{c}\text { Antena } 3 \\
\text { (Lunes, } 21 \text { de octubre } \\
\text { de 2013) }\end{array}$} & \multicolumn{2}{|c|}{$\begin{array}{c}\text { Telecinco } \\
\text { (Lunes, } 21 \text { de } \\
\text { octubre de 2013) }\end{array}$} \\
\hline & & & Importe & $\begin{array}{l}\psi \text { w sobre al } \\
\text { valor total }\end{array}$ & Importe & $\begin{array}{l}\text { \% sobre el } \\
\text { valor total }\end{array}$ \\
\hline 3 & SONY & $16.789,12$ & 0,00 & $0,00 \%$ & 25,00 & $0,13 \%$ \\
\hline 4 & MUTUA MADRILEÑA & $7.397,17$ & 16,80 & $0,23 \%$ & 14,00 & 0,198 \\
\hline 6 & ORANGE & $11.988,38$ & 0,00 & $0,00 \%$ & 25,00 & $0,21 \%$ \\
\hline 7 & PEUGEOT & $7,188,70$ & 2,55 & $0,04 \%$ & 151,90 & $2,11 \%$ \\
\hline 8 & MERCEDES & $3.453,52$ & 27,30 & $0,79 \%$ & 20,00 & $0,58 \%$ \\
\hline
\end{tabular}

Fuente: Arce Media-Dragón/Elaboración propia

No debemos olvidar además, que RTVE no ha podido actuar en ningún momento como contrapeso al dominio abrumador del duopolio. El último año que dispuso de publicidad (año 2009) llegó casi a disponer del $20 \%$ del mercado.

Aunque no exista de facto una correlación evidente entre la creación de este duopolio televisivo y la deriva de RTVE $-\mathrm{y}$ a falta de un estudio más pormenorizado sobre el particular-, sí puede concluirse, y así lo señaló también el director técnico del Estudio de la Inversión Publicitaria en España 2011, de Infoadex4, que ha existido un trasvase entre la publicidad que comercializaba la cadena estatal y las dos grandes cadenas privadas, como ha observado incluso la propia CNC. Un hecho innegable es que, tras dotarse del nuevo marco financiero y comenzar las restricciones en su financiación y variar el contenido de sus informativos, TVE ha bajado por vez primera en su historia por debajo del $10 \%$ de la audiencia total, quedando relegada cada vez más a ejercer un papel testimonial en el ámbito televisivo y, sobre todo, en el aspecto informativo.

\section{Conclusiones}

Las dos grandes plataformas televisivas disponen actualmente de 17 canales de televisión, con casi el $90 \%$ de la contratación publicitaria en abierto, el $57,9 \%$ de la audiencia, y entre ambas suponen un $50 \%$ del total de la publicidad contratada en medios convencionales. Junto con los grandes players de Internet -fundamentalmente, Google- dominan ampliamente el mercado publicitario de medios convencionales. La consecuencia inmediata es que suponen una rémora para la supervivencia de otros canales de televisión o la entrada de nuevos concurrentes en el mercado, ya sea en forma de start up periodísticas o audiovisuales y nuevas iniciativas de VOD, y acaparan además una buena parte de los contenidos Premium.

El dominio ejercido por ambas plataformas ha tenido también una incidencia muy directa en el declive de RTVE y de las cadenas autonómicas. En el primero de los casos, por la retirada de la publicidad y su ausencia en la puja de contenidos de retransmisión deportiva, y en el segundo, porque han visto como disminuía su facturación publicitaria.

Hoy día en España es imposible para los grandes anunciantes -distribución, automoción, banca- llegar a su público objetivo en cualquier lugar de España sin el concurso de los canales de ambas plataformas. Y en cuanto al mercado de prensa, una vez desparecida la ventaja que poseían los editores de diarios sobre los anunciantes 0 , dicho de otra manera, una vez que se ha

\footnotetext{
4 Según Pedro Villa, "los 422 millones de euros que ingresó TVE en publicidad en 2009 claramente se han quedado en 2010 en las grandes cadenas de televisión, por lo que los principales grupos de televisión han visto aumentar sus ingresos publicitarios" (Europa Press, 28 de febrero de 2011).
} 
producido la disminución del poder que estos últimos habían detentado históricamente hasta la irrupción de Internet, los anunciantes han adquirido un enorme poder. Ya no es solo que hayan desaparecido los abundantes márgenes de los buenos tiempos, sino que incluso esta tendencia encuentra su aliado natural en la publicidad barata que se realiza en la red. Ahora bien, y dado que a la televisión generalista y en abierto todavía le queda una larga vida, los grandes anunciantes no ven de momento manera alguna de contrarrestarla ni de imponer sus condiciones a las grandes cadenas televisivas.

\section{Referencias bibliográficas}

Aguirre Madrid, F. y González Jansina, J.P. (2012): "Medios de comunicación y publicidad privada. Comentarios desde los estándares internacionales de los derechos humanos". Comunicación y medios, 25, Instituto de la Comunicación e Imagen. Universidad de Chile, pp. 91-103.

Alcolea Díaz, G. (2003): La televisión digital en España. Sevilla: Comunicación Social.

---- y Pérez Serrano, M. J. (2013): "Análisis de las recientes actuaciones de la Comisión Nacional de la Competencia (CNC) sobre el sector audiovisual". En García Santamaría, J.V. (edic.): Concentración y pluralismo en los medios de comunicación españoles. La Laguna: Cuadernos Artesanos de Comunicación, $\mathrm{n}^{0}$ 38, pp. 59-88.

Almirón, N. et al (2012): "External Pluralism Protection in five EU Countries and the US: The Regulatory Authorities'Views". OBS Journal, vol. 6, 129-157.

Álvarez Monzoncillo, J. M. (2011): La televisión etiquetada: nuevas audiencias, nuevos negocios. Barcelona: Ariel/Fundación Telefónica.

Anderson, C.W., Bell, E. y Shirky, C. (2013): Periodismo postindustrial: adaptación al presente. Huesca: eCícero.

Arce Media (2013): "Ranking inversión octubre 2013". Disponible en: http://www.arcemedia.es/noticia_informe_view.aspx?id=4279.

Arnanz, C.M. (2002): Negocios de televisión. Transformaciones del valor en el modelo digital. Barcelona: Gedisa Editorial.

Artero, J.P. (2008): El mercado de la televisión en España: oligopolio. Barcelona: Deusto.

Barlovento Comunicación (2013): El comportamiento de la audiencia televisiva: octubre 2013. Disponible en: http://www.barloventocomunicacion.es/images/publicaciones/INFORME\%20AUDIENCI AS\%20BARLOVENTO\%20-\%200CTUBRE\%202013.pdf.

Bustamante, E. (2009): "Un análisis consolidado de análisis sobre el futuro audiovisual digital" (prólogo). En Francés i Doménec, M. (edic.): Hacia un nuevo modelo televisivo. Barcelona: Gedisa.

Campos Freire, F. (2012): "Modelos de financiación de las televisiones públicas autonómicas". En Miguel de Bustos, J.C. y Casado del Río, M.A. (edic.): Televisiones autonómicas. Evolución y crisis del modelo público de proximidad (pp. 143-172). Barcelona: Gedisa.

Chan-Olmsted, S. M. (1998): "Mergers, Acquisitions, and Convergence: The Strategic Alliances of Broadcasting, Cable Television, and Telephone Services". The Journal of Media Economics, 11(3), 3346.

Council of Europe (2004): "Transnational media concentrations in Europe". Strasbourg: Media Division, Doctorate General of Human Rights. Doyle, G. (2003): Media Ownership. London: Sage.

Europa Press (28 de febrero de 2011): "La inversión en mecenazgo, actos de patrocinio y RSC creció 
en 2010 un $7,4 \%$, según Infoadex". Disponible en: http://www.europapress.es/epsocial/rsc/noticia-inversion-mecenazgo-actos-patrocinio- rsc-crecio-201074-infoadex-20110228122420.html.

Faustino, P. (2010): Pluralismo, concentraçao dos media e cidadania. Lisboa: Media XXI/Formal Press.

Fernández Fernández, F. (2013): "Descapitalización, corto plazo y pluralismo: la crisis económica y el marco regulatorio de la UE propician la concentración mediática en los países periféricos": Santiago de Compostela. Actas en edición Congreso Ibercom 2013.

García Castillejo, A. (2009): "Pluralidad y concentración en el sistema audiovisual español". En Francés i Doménec, M. (edic.): Hacia un nuevo modelo televisivo (pp. 129-140). Barcelona: Gedisa.

García Santamaría, J. V. (2013): "Televisión y concentración en España: el duopolio de Mediaset y Atresmedia". Palabra Clave 16 (2), 366-397.

--- y Fernández-Beaumont, J. (2012): "Concentración y pluralismo en el nuevo escenario audiovisual español. Análisis de la absorción de la cadena Cuatro por Telecinco". Análisi, nº 47, p. 47-54.

George, L. (2007): "What's fit to print: the effect of ownership concentration on product variety indaily newspaper markets". Information Economics and Policy, 19(3-4), 285-303.

Infoadex (2013): "Estudio Infoadex de la inversion publicitaria en España en 2013. Correspondientes al ejercicio 2012". Disponible en: www.infoadex.es.

Lanza, E. y Buquet, G. (2011): La televisión privada comercial en Uruguay. Caracterización de la concentración de la propiedad, de las audiencias y la facturación. Montevideo: Fundación FriedrichEbert-Stitung.

Llorens, C. (2010): "Spain's Media Concentration Policy: A Patchwork Crucial to the Understanding of the Spanish Media System". International Journal of Communication, 4, pp. 844-864.

Miguel de Bustos, J.C. (2007): "Cambios estructurales en marcha. Los grupos de comunicación españoles". En Bustamante, E. (edic.): Cultura y Comunicación para el siglo XXI (pp. 185-213). Tenerife: Ideco.

Mosco, V. (1996): Political Economy of Communication: Rethinking and Renewal. Sage: London.

Peltier, S. (2004): "Mergers and Acquisitions in the Media Industries: Were Failures Really Unforeeable?", en The Journal of Media Economics, 17(4), 261-178.

Pérez Gómez, A. (2002): El control de las concentraciones de medios de comunicación. Madrid: Dykinson.

Rodríguez Pardo, J. (2005): "Concentraciones mediáticas en España y pluralismo informativo: una búsqueda de los criterios informativos de control de la Comisión Europea (1990- 2004). Sphera pública: revista de ciencias sociales y de la comunicación, 5, pp. 109-132.

Sánchez-Tabernero, A. (2010): "Los grupos de comunicacion en España: estrategias frente a la crisis". En Campos Freire, F. (coord.): El cambio mediático. Sevilla/Zamora: Comunicación Social.

Segovia, A.I. (2001): "Aviso para navegantes. Concentración y privatización de las comunicaciones en Estados Unidos". En Quirós, F. y Sierra, F.: Crítica de la Economía Política de la Comunicación y la Cultura. Sevilla: Comunicación Social. 
Tomás Olalla, F. (2004): "Revisión crítica de las relaciones entre pluralismo y mercado en los medios audiovisuales". Zer, n 16 , pp. 45-56.

Vertele (2013): "Grandioso estreno de 'El tiempo entre costuras' con 5 millones y $25.5 \%$ de share". Disponible en: http://www.vertele.com/noticias/el-tiempo-entre-costuras- deslumbra-y-le-hace-un-roto-ala-voz-e-isabel/.

Zallo, R. (2011): Estructuras de la comunicación y la cultura. Barcelona: Gedisa 